\title{
Avaliando o Desempenho Discente em um AVA: Um Estudo de Caso Utilizando Estatística Multivariada
}

\author{
Thiago I. A. Souza ${ }^{1}$, Artur O. R. Franco ${ }^{2}$, \\ Thomaz E. V. Silva ${ }^{3}$, F. Herbert. L. Vasconcelos ${ }^{3}$ \\ ${ }^{1}$ Departamento de Física - Universidade Federal do Ceará (UFC) \\ Caixa Postal 60020-181 - Fortaleza - CE - Brasil \\ ${ }^{2}$ Instituto de UFC Virtual - Universidade Federal do Ceará (UFC)
}

${ }^{3}$ Departamento de Engenharia de Teleinformática - Universidade Federal do Ceará (UFC)

\{thiagoiachiley, artur.fhtagn\}@gmail.com, \{thomazveloso, herbert\}@virtual.ufc.br

\begin{abstract}
The evaluation of learning is not restricted to the quantitative measurement of student learning. Highlights some factors that can be analyzed during the teaching process: the influence of the teacher, the school environment, the course curriculum; evaluative metrics, among others. These factors include the structure of the course curriculum, as the object of study of this research. Thus, this study aims to analyze the curriculum of an undergraduate course in blended mode. In this context, a qualitative study from a quantitative analysis is proposed to identify the possible relationship between the disciplines of a course and infer how the curriculum for a course can influence the evaluation parameters. Results show the effectiveness of the application of multivariate statistical techniques in the analysis and understanding of educational processes.
\end{abstract}

Resumo. A avaliação da aprendizagem não se restringe apenas a aferição quantitativa da aprendizagem discente. Destacam-se alguns fatores que podem ser analisados durante o processo de ensino: a influência do docente; o ambiente escolar; o currículo do curso; métricas avaliativas; dentre outros. Dentre esses fatores destacamos a estrutura curricular do curso, como sendo objeto de estudo desta pesquisa. Desta forma, o presente trabalho tem como objetivo analisar o currículo de um curso de graduação na modalidade semipresencial. Neste contexto, um estudo qualitativo a partir de uma análise quantitativa é proposto para identificar as possíveis relações existentes entre as disciplinas de um curso e inferir como o currículo de um curso pode influenciar nos parâmetros da avaliação. Os resultados alcançados demonstram a eficácia da aplicação de técnicas da estatística multivariada na análise e compreensão de processos educacionais.

\section{Introdução}

A educação a distância (EaD), por intermédio dos avanços das tecnologias da informação e comunicação, tem viabilizado a realização e aplicação de diferentes metodologias educacionais com o objetivo de potencializar a aprendizagem discente. Com 
essa nova tendência educacional, a avaliação discente tem se tornando esse um dos temas mais questionados e estudados na atualidade pelos pesquisadores da área.

De acordo com Sevindik (2010), a EaD pode ser definida como um processo de ensino-aprendizagem estabelecido através de uma dupla interação entre o professor e o aluno, que mediados por tecnologias, estão separados espacial e/ou temporalmente. A partir do desenvolvimento destas tecnologias, práticas de educação a distância são aplicadas aos métodos convencionais de ensino, que seguindo essa evolução de perto, trouxe consigo a temática de como avaliar os resultados obtidos no processo de ensinoaprendizagem Iskenderoglu (2012) e Karal (2010).

Para Sevindik (2010), o processo de avaliação é descrito como um feedback nas tomadas de decisões para indicar se um conceito de educação é eficaz ou não, sendo assim, considerado como a chave mais importante para o sistema de ensino moderno. Tal feedback tem como finalidade não apenas certificar os discentes, mas também, rever e melhorar as ferramentas de avaliação Iskenderoglu (2012). Desta forma entendemos o processo de avaliação como parte integrante de um conjunto de componentes inter-relacionados que influenciam o resultado da aprendizagem, tais como, o método de ensino, a estrutura do curso, ambiente escolar, o currículo das disciplinas, dentre outros.

Essas percepções inerentes a avaliação e a forma de como avaliar também influenciaram a $\mathrm{EaD}$, mediante o uso freqüente de instrumentos de verificação quantitativa de desempenho David (2007). Porém para Vasconcelos (2011), o processo de avaliação ultrapassa os limites de uma simples mensuração de rendimento discente, deixando de ter um caráter simplesmente classificatório para o estabelecimento de padrões, identificação de transformações e aquisições de conhecimentos de novas aprendizagens, por meio de um processo pelo qual se pode determinar em que medida ou intensidade tais mudanças de fato ocorrem. Nessa perspectiva os Ambientes Virtuais de Aprendizagem (AVAs) favorecem um controle maior das interações entre alunos e professores, permitindo assim uma organização das informações coletadas. Essa diversidade de informações registradas no AVA pode ser adequadamente analisada através de métodos de análise de mineração de dados Almeida e Pimentel (2010), especialmente da estatística multivariada.

Pesquisas como as realizadas por Wright, Horn e Sanders (1997), têm mostrado objetivamente a influência de alguns dos fatores integrantes do processo de avaliação no âmbito do contexto da aprendizagem através da utilização de modelos estatísticos mistos, que possibilitam vislumbrar a exploração de diferentes padrões de análise desses fatores.

O presente trabalho tem como objetivo analisar um dos fatores que integra o processo de avaliação e influencia na aprendizagem discente, o currículo de um curso de graduação na modalidade semipresencial. Inicialmente, o artigo abordará estudos que tem em seu eixo central a temática da avaliação em AVAs. Em seguida, é descrita um pouco da ferramenta estatística multivariada que tratou os dados coletados do AVA. Por conseguinte, a metodologia do trabalho é detalhada, especificando os detalhes inerentes a essa pesquisa. Na conclusão, discutimos a proposta de validação da estrutura curricular do curso em estudo, destacando a potencialidade da ferramenta estatística em seu uso no contexto educacional. 


\section{O Processo de Avaliação da Aprendizagem em AVA}

Com o crescimento da utilização dos ambientes virtuais de aprendizagem (AVA) em diversas instituições de ensino, Sales (2008) e Vasconcelos (2008) abordam o desenvolvimento de ferramentas que tem com objetivo, melhorar o processo de avaliação da aprendizagem. Stricker (2011) utiliza o AVA como uma ferramenta de auto-avaliação com o objetivo de aumentar o processo de aprendizagem dos alunos. Piconez (2011), analisa e aponta o potencial inovador do AVA caracterizando a sua relevância para propostas pedagógicas vislumbrando o aperfeiçoamento do processo de avaliação e acompanhamento da aprendizagem em ambientes virtuais.

Devido a grande importância de entender as práticas avaliativas e seu delineamento, o conceito de avaliação vem sendo muito discutido nos últimos anos por pesquisadores da área. Para David et al (2007), a avaliação é considerada como um processo cuja finalidade é o diagnóstico de problemas para auxílio na tomada de decisão e identificação de novos rumos. Já Anacleto (2011), define a avaliação da aprendizagem como um elemento fundamental do processo educativo, onde as novas formas de ensinar devem trazer consigo novas metodologias avaliativas, de forma a subsidiar e assegurar o mesmo nível de aprendizagem dos métodos tradicionais.

Apesar de haver uma busca cada vez mais intensa para se explorar as vertentes de otimização do processo de avaliação, sua aplicação tem que ser pensada levando-se em conta o contexto em que se dá o processo da aprendizagem, sobretudo quando tratamos do ensino na modalidade semipresencial. Nessa perspectiva Bernini (2012) considera a escolha do processo avaliativo como um desafio a ser enfrentado, haja vista que, os objetivos inseridos nesse processo avaliativo devem estar alinhados aos objetivos de ensino. David et al (2007) complementa enfatizando também que a definição dos critérios de avaliação é um processo mutável que está sujeito às necessidades dos sujeitos envolvidos no processo de ensino/aprendizagem. Em especial, Bassani e Behar (2009) aponta para o fato de que a definição de critérios de avaliação coerentes tem sido uma das dificuldades de maior destaque na modalidade de ensino a distância.

Por oferecer e disponibilizar ao corpo docente e discente acesso e registro de suas ações em suas diferentes dimensões diagnósticas, somativas e formativas, o processo de avaliação em AVA se torna inovador. De acordo com Bassani (2006), a avaliação em AVA se dá a partir de três perspectivas: avaliação por meio de testes online; avaliação da produção individual dos estudantes e análise das interações entre os alunos. Entretanto, Bassani e Behar (2009) contestam a utilização de testes online, pois apresentam limitações de acordo com a concepção da nota como base no produto final do conhecimento, sem levar em consideração o processo de construção de conceitos por parte dos discentes. Ademais, existem dificuldades no que diz respeito a consideração de uma avaliação adequada para as atividades coletivas. Alguns esforços foram feitos nessa direção, como os trabalhos de Sales et al (2008) e Vasconcelos (2008), que desenvolveram métodos e ferramentas com a finalidade de avaliar a construção de conhecimentos por parte dos alunos, levando-se em conta o ambiente dinâmico de atividades interativas nos AVAs.

Com a possibilidade de se registrar informações de cunho individual relacionadas a frequiência, assiduidade e desempenho dos alunos, bem como também, informações de caráter coletivo relacionadas às interações estabelecidas entre os participantes de uma determinada atividade ou curso Bassani (2006), a dinâmica de dados dentro desses AVAs 
passou a ser investigada. Nessa perspectiva, métodos da estatística multivariada passaram a ser utilizados no contexto da educação para o tratamento dessas informações, afim de se observar um padrão no comportamento desses dados e estabelecer inferencias acerca das análises vislumbradas e entender cada vez mais acerca dos processos avaliativos e seu desdobramento no contexto da EaD.

\section{Estatística Multivariada para Seleção de Características}

\subsection{Análise Fatorial}

A análise fatorial é um método proveniente da estatística multivariada que tem como ideia básica reexpressar um determinado conjunto de dados com $\mathrm{n}$ variáveis em termos de um número menor de variáveis latentes (também chamada de fatores), que representam subjetivamente, segundo a visão do especialista, as dependências entre as variáveis observadas Katsikatsou et al (2012) e Green (2011). Seguindo essa definição, o objetivo principal da análise fatorial é explorar as correlações existentes entre as variáveis diversas de um problema. Segundo Katsikatsou et al (2012), a análise fatorial pode ser segregada em duas peculiares modalidades de análises: análise confirmatória e exploratória.

$\mathrm{Na}$ análise confirmatória, o foco principal é viabilizar testes de qualidade de ajuste para os modelos, podendo ainda ser utilizada para avaliar a significancia estatística destes. Desta forma, a análise fatorial confirmatória é realizada para verificar a confirmação de uma estrutura pré-determinada. Na análise exploratória, a ideia básica é encontrar um novo conjunto de dados de baixa dimensionalidade formado pelas variáveis não observadas, denominadas de variaveis latentes ou fatores comuns, de tal forma que venham a representar o máximo de covariância ou correlação entre as variáveis originais.

A análise fatorial exploratória, que é a análise que utilizamos nesta pesquisa, é utilizada com o propósito de examinar e descrever padrões nas variâncias ou correlações de um conjunto de dados com múltiplas dimensões. Neste segmento, esta análise busca a investigação das estruturas de correlação, muito se assemelhando, neste aspecto, ao método de análise das componentes principais (PCA), divergindo apenas na representatividade das variáveis originais. Dessa forma, a medida que na análise fatorial as variáveis originais são representadas pela combinação linear das novas variáveis (fatores latentes), por outro lado no PCA, as novas variáveis (componentes principais) são as combinações lineares das variáveis originais Green (2011).

A análise fatorial pode ser escrita na forma de um modelo matemático que descreve o tratamento dado aos dados originais em estudo. Desta forma a variável formada $\mathrm{X}$ representa um vetor com $x_{p}$ variáveis originais, tendo como objetivo fazer com que a variância observada em cada medida deste conjunto possa ser atribuída a um número relativamente reduzido de fatores comuns, temos:

$$
Y=\left(y_{1}, \ldots, y_{k}\right)^{\prime}
$$

em que estes fatores representariam as variáveis latentes, tendo desta forma um conjunto de dados menor que o original $(k<p)$, porém com representatividade do todo, contendo essencialmente a mesma informação.

\subsection{Análise de Componentes Principais (ACP)}

Os dois métodos analíticos usados nesta pesquisa, AF e ACP, embora sejam semelhantes apresentam distintos fundamentos teóricos de abordagem. A análise de com- 
ponentes principais, que será discutida nesta subseção, é uma técnica multivariada que tem como objetivo alcançar a redução de dimensionalidade de um conjunto de dados com $\mathrm{k}$ dimensões, para um número menor de $\mathrm{p}$ dimensões independentes, onde $p<k$. Lay (2007) destaca ainda que, além da redução de dimensionalidade, a ACP tem por finalidade a identificação de relações entre variáveis discretas através de uma transformação linear dessas variáveis em um conjunto de atributos descorrelacionados, de modo que os dados resultantes tenham suas componentes mais relevantes nas primeiras dimensões, denominadas componentes principais.

Para Green (2011), ao contrário da AF, a ACP além de reduzir dimensionalidade, está preocupada com a distribuição da variabilidade das informações nas variáveis originais. Entende-se então que a ACP é uma extensão da AF.

A característica geral da ACP está em expressar as dimensões reduzidas (novos atributos) como uma combinação linear das dimensões originais (dados originais). Desta forma, o modelo matemático que representa a escolha das componentes principais é:

$$
Z=A x
$$

onde a unidade de medida dessas novas componentes $(Z)$ geradas são combinações lineares não correlacionadas, podendo desta forma, identificar o grau de contribuição de cada variável em relação a variância total dos dados originais. Devido à construção do modelo, a primeira componente, que captura a maior fração de variância dos dados, é sempre a mais representativa, e as demais hierarquicamente organizadas de forma descendente de seus respectivos autovalores, assumem os menores valores de variância, possuindo consequentemente menor representatividade.

\section{Metodologia}

\subsection{Caracterização dos Dados Coletados}

Neste estudo, uma investigação qualitativa a partir de uma análise quantitativa foi realizada através da coleta de dados que ocorreu por meio das notas obtidas por 126 alunos de um curso de Licenciatura em Física, na modalidade semipresencial, de uma Universidade localizada no nordeste brasileiro. O desempenho dos 126 discentes foi avaliado em um Ambiente Virtual de Aprendizagem na Internet ao longo de 6 disciplinas, que formam o primeiro ano do curso em estudo.

\subsection{Disciplinas Analisadas}

As disciplinas analisadas abordam conteúdos referentes ao ciclo básico da Física. A Tabela 1 apresenta as disciplinas que foram cursadas pelos alunos nos dois semestres iniciais do curso.

\subsection{Ferramenta Computacional}

Como procedimento na realização da análise dos dados coletados, foi utilizado o software Statistical Package for the Social Sciences(SPSS), que é um sistema de análise estatística em um ambiente gráfico de manuseamento de dados, recomendado para a análise de fenômenos ou processos sociais, econômicos, psicológicos e educacionais SPSS (2007). 


\begin{tabular}{|c|c|}
\hline Semestre & Disciplinas \\
\hline $1^{\circ}$ & $\begin{array}{c}\text { Física 1 } \\
\text { Laboratório de Física 1 } \\
\text { Matemática 1 }\end{array}$ \\
\hline $2^{\circ}$ & $\begin{array}{c}\text { Física 2 } \\
\text { Laboratório de Física 2 } \\
\text { Informática Aplicada à Educação }\end{array}$ \\
\hline
\end{tabular}

Tabela 1. Disciplinas Analisadas.

Fazendo uso do pacote de análise fatorial do SPSS, que tem como objetivo identificar parâmetros subjacentes, ou fatores, responsáveis por explicar os padrões de correlações existentes intrinsecamente no conjunto de variáveis observadas, foram determinados os fatores mais relevantes para a análise Lay (2007). Utilizamos o método da análise de componentes principais como a técnica de extração dos fatores.

\section{Resultados e Discussão}

\subsection{Validando a Base de Dados}

Afim de determinar a confiabilidade e avaliar a consistência interna dos atributos para a bases de dados analisada, utilizamos o alfa de Cronbach. O valor de alfa nos rendeu uma alta confiabilidade dos dados obtidos (alfa de Cronbach $=0,823$ ), garantindo assim uma forte estimativa da consistência interna das variações dos itens para a análise, Cortina (1993).

\subsection{Análise dos Fatores}

Para se iniciar uma análise fatorial, é necessário que seja feito alguns testes à matriz do conjunto de dados, afim de investigar a eficácia da utilização do método. Para tanto, utilizamos os testes de Esfericidade de Bartlett e a Medida de Adequacidade da Amostra de Kaiser-Meyer-Olkin (KMO) para analisar a estrutura de correlação dos dados quanto a sua adequação ou não à aplicação da análise fatorial Moita-Neto (2009).

O teste de Kaiser-Meyer-Olkin (KMO), examina o ajuste dos dados tomando todas as variáveis simultaneamente e provê uma informação sintética sobre os dados, indicando a proporção da variância dos dados que pode ser considerada comum a todas as variáveis. Desta forma, quanto mais próximo de 1 (unidade) melhor o resultado. Já o teste de esfericidade de Bartlett, testa a hipótese de que a matriz de correlação é uma matriz identidade, isto é, que não há correlação entre as variáveis. Dessa forma, procura-se para um nível de significância assumido em 5\% rejeitando a hipótese nula de matriz de correlação identidade.

Os valores retornados por esses testes no presente estudo, sugerem que o uso da análise fatorial é apropriado para analisar a matriz de correlação referente às disciplinas cursadas pelos alunos, permitindo assim, a efetivação da utilização do método.

Na Tabela 2, são apresentados os valores dos testes, onde $K M O>0,5$ e Bartlett com rejeição de hipótese nula Moita-Neto (2009). Com a aplicação dos testes, constatouse que os dados são adequados a análise. 


\begin{tabular}{|l|c|}
\hline $\begin{array}{l}\text { Adequação da } \\
\text { Amostra - KMO }\end{array}$ & Esfericidade de Bartlett \\
\hline 0,734 & 396,877 \\
\hline
\end{tabular}

Tabela 2. Teste KMO e Bartlett.

\subsection{Seleção das Componentes Principais}

Existem vários critérios para se determinar quantas componentes devem ser analisadas pelo modelo. Dentre elas está o percentual da variância total explicada por cada componente associada ao seu respectivo autovalor. A análise efetuada para seleção das componentes principais considerou os valores absolutos e acumulados das respectivas porcentagens de variação dos dados explicados para cada novo vetor incorporado na análise (Tabela 3).

O Teste Scree (Figura 1), que tem como finalidade validar a metodologia para a seleção das componentes, é um gráfico dos autovalores em função da ordem das componentes principais, representando graficamente a porcentagem de variância explicada por componente. Quando esta porcentagem se reduz e a curva passa a ser quase paralela ao eixo das abcissas, as componentes correspondentes podem ser desprezadas. É importante destacar que as componentes obtidas nesta análise constituem as novas variáveis que serão utilizadas nas análises subseqüentes do estudo.

\begin{tabular}{|l|c|c|c|}
\hline & \multicolumn{3}{|c|}{ Autovalores Iniciais } \\
\hline Componentes & Total & \% Variância & \% Acumulativa \\
1 & 3,212 & 53,534 & 53,534 \\
$\mathbf{2}$ & $\mathbf{1 , 4 1 9}$ & $\mathbf{2 3 , 6 4 3}$ & $\mathbf{7 7 , 1 7 7}$ \\
3 & 0,502 & 8,364 & 85,541 \\
4 & 0,453 & 7,542 & 93,083 \\
5 & 0,282 & 4,699 & 97,782 \\
6 & 0,133 & 2,218 & 100,00 \\
\hline \hline
\end{tabular}

Tabela 3. Variância Explicada dos Dados.

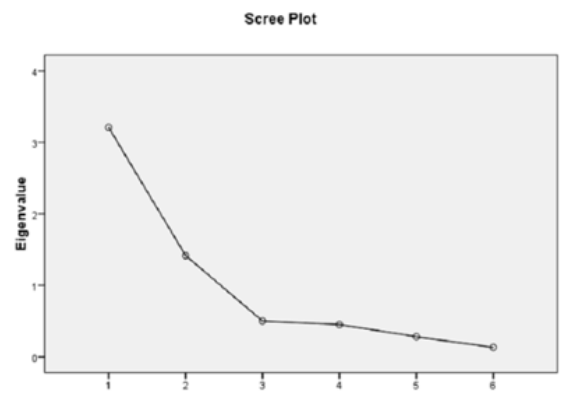

Figura 1. Gráfico Scree para os autovalores referentes aos Alunos.

Observando o comportamento da inclinação da curva do Gráfico Scree através da Figura 1, percebemos uma inflexão brusca da curva até se estabilizar em um dado ponto do gráfico. Nesta análise observa-se este decaimento acentuado até a terceira componente. 
Assim, o número escolhido de Componentes Principais foi baseado no critério de truncamento de Kaiser Moita-Neto (2009), que considera como mais significativos os autovalores cujos valores sejam superiores à unidade.

A ACP aplicada aos dados iniciais que apresentavam 6 parâmetros principais, inerentes às disciplinas, reduziu para 2 componentes principais os atributos analisados, as quais representam uma variância explicada de 77,177\% dos dados originais.

\subsection{Análise das Componentes Principais}

Como resultados da aplicação da Análise de Componentes Principais na matriz das correlações investigada podem vislumbrar de forma significativa como se deram os padrões de comportamento das disciplinas inerentes ao primeiro ano do curso, ao longo das duas componentes principais selecionadas.

A partir da análise dos resultados retornados pelo método observamos, através da plotagem dos fatores de carregamento (Figura 2), a formação de dois agrupamentos que apresentam um padrão na disposição dos atributos.

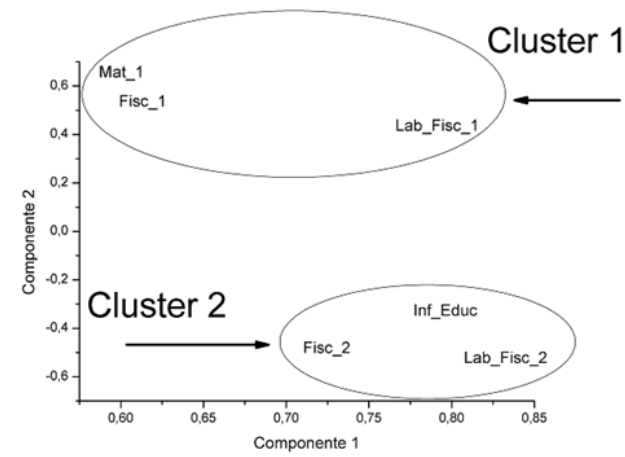

Figura 2. Distribuição das Disciplinas no Espaço das Componentes Principais.

Observando a formação dos dois agrupamentos na figura acima, vislumbramos a disposição das disciplinas inerentes ao primeiro ano do curso. O primeiro grupo (Cluster 1) indica a presença das disciplinas inerentes ao primeiro semestre do curso, possuindo elevados fatores de carregamento para a segunda componente, enquanto que o segundo agrupamento (Cluster 2) indica o agrupamento inerente às disciplinas do segundo semestre, com elevados fatores de carga para a primeira componente.

Os elevados valores de carregamento para a componente 2, formando o Cluster 1, pode ser explicado devido a semelhança entre as três disciplinas, haja vista que ambas requerem do discente uma expertise mais aguçada quanto ao uso do formalismo matemático.É interessante notar que o padrão se mantém na disposição dos fatores na componente 1, formando o Cluster 2. Além de agrupar os atributos, a ACP também nos permite nomear essas duas componentes, tornando possível a interpretação do significado das componentes 1 e 2 . A componente 1 pode ser interpretada como a relação entre as disciplinas introdutórias ao curso, ao passo que a componente 2 , relaciona um grupo de disciplinas que corrobora com o primeiro grupo, formando assim o ciclo básico do primeiro ano do curso em estudo. 


\section{Considerações Finais e Perspectivas Futuras}

Este trabalho apresenta uma significativa contribuição da aplicação de uma técnica da estatística multivariada, o método ACP, em dados educacionais inerentes ao processo de avaliação da aprendizagem, com ênfase nos fatores relacionados a avaliação, em específico, a análise sobre a estrutura curricular de um curso de graduação no âmbito do ensino de física.

Os resultados dos testes KMO e de esfericidade de Bartlett apontam para a validação da aplicação da técnica na base de dados selecionada. A partir da análise da plotagem das cargas podemos verificar os padrões de disposição na localização das disciplinas nas componentes selecionadas, inferindo sobre a importância da investigação dos grupos formados através da análise dos agrupamentos.

Com a aplicação do ACP percebemos uma homogeneidade na formação dos dois grupos. No Cluster 1, verificamos uma maior proximidade entre as disciplinas teóricas, Matemática 1 e Física 1, ressaltando a discrepância para com o Laboratório de Física 1, dado ao fato de ser uma disciplina de caráter experimental. Já para o Cluster 2, verificamos uma menor variabilidade na disposição dos três atributos, indicando que nenhuma disciplina se sobressai da outra, possuindo os mesmos níveis de importância.

Neste presente estudo de caso, a partir do desempenho discente investigado, podemos conhecer as relações existentes entre as disciplinas abordadas, validando desta forma, a estrutura curricular prévia adotada pela coordenação do curso em análise.

Como trabalho futuro sugere-se uma análise mais minuciosa na plotagem dos fatores de carregamento bem como na análise dos agrupamentos, explorando outros métodos de identificação dos grupos, e a aplicação de outras técnicas de análise de dados que trabalham com dimensões de ordem superior a dois Kroonenberg (2008).

\section{Referências}

ALMEIDA, A. e PIMENTEL, E. P. (2010). Mineração de dados no ensino a distância para fins de avaliação do uso das ferramentas de aprendizagem do ambiente tidia-ae. In: Anais do III WAvalia.

ANACLETO, G. M. C. (2011). Avaliação da Aprendizagem em Educação a Distância. Anais do XXII SBIE - XVII WIE, IV WAvalia.

BASSANI, P. S. (2006). Mapemamento das interações em ambiente virtual de aprendizagem: uma possibilidade para avaliacão em educacão a distância. Tese (Doutorado). PhD thesis, Universidade Federal do Rio Grande do Sul - PPGIE, Porto Alegre.

BASSANI, P. S. e BEHAR, P. A. (2009). Modelos Pedagógicos em Educação a Distância. Avaliação da Aprendizagem em Ambientais Virtuais. Artmed.

BERNINI, D. S. D. (2012). Objetivos procedimentais, atitudinais e conceituais na avaliação da aprendizagem. Anais do XXIII SBIE - XVIII WIE, V WAvalia.

CORTINA, J. M. (1993). What is coefficient alpha? An examination of theory and application. Journal of Applied Psychology, 78, 98-104.

DAVID, P. B., PEQUENO, M. C., da SILVA, A., SOUSA, C. F., JÚNIOR, G. S. V., de CASTRO FILHO, J. A., VENTURA, P. P. B. e MAIA, S. (2007). Avaliação da aprendi- 
zagem em educação a distância numa perspectiva sócio-interacionista. Anais do XVIII Simpósio Brasileiro de Informática na Educação.

GREEN, P. E. (2011). Multivariate Data Analysis. Cengage Learning.

ISKENDEROGLU, M., ISKENDEROGLU, T. A., PALANCI, M. (2012). Opinion of Teaching Staff in Distance Education Systems, Regarding the Assessment and Evaluation Process. Procedia Social and Behavioral Sciences 46: 4661-4665.

KARAL, H., CEBIL, A., PEKSEN, M. (2010). Student opinions about the period of measurement and evaluation in distance education: the difficulties. Procedia Social and Behavioral Sciences 9: 1597-1601.

KATSIKATSOU, M., MOUSTAKI, I. e YANG-WALLENTIN F., JORESKOG K.G. (2012). Pairwise likelihood estimation for factor analysis models with ordinal data. Computational Statistics and Data Analysis 56: 4243-4258.

KROONENBERG, P. M. (2008). Applied Multiway Data Analysis. Wiley.

LAY, D. C. (2007). Álgebra Linear e Suas Aplicações, $2^{\circ}$ ed. LTC.

MINGOTI, S. A. (2005). Análises de Dados Através de Métodos de Estatística Multivariada.

MOITA-NETO, J. M. (2009). Estatística Multivariada na Pesquisa, volume 5. Sapiência (FAPEPI).

PICONEZ, S. C. B. (2011). Avaliação em Ambientes Virtuais de Aprendizagem: articulação dialética de suas dimensões. Anais do XXII SBIE - XVII WIE, IV WAvalia.

SALES, G. L., BARROSO, G. C. e SOARES, J. M. (2008). O indicador de aprendizagem learning vectors como instrumento automatizado de avaliação para suporte a aprendizagem em EAD. Anais do XXVIII Congresso da SBC.

SEVINDIK, T., COMERT, Z. (2010). Using algorithms for evaluation in web based distance education. Procedia Social and Behavioral Sciences 9: 1777-1780.

STRICKER, D., WEIBEL, D., WISSMATH, B. (2011). Efficient learning using a virtual learning environment in a university class. Computers and Education 56: 495-504.

WRIGHT, P., HORN, S. P. e SANDERS, W. L. (1997). Teacher and Classroom Context Effects on Student Achievement: Implications for Teacher Evaluation. S. Journal of Personnel Evaluation in Education 11: 57-67.

VASCONCELOS, F. H. L. (2008). Avaliação sócio-interacionista aplicada ao contexto da ead em cursos de graduação semi-presenciais mediado por um ambiente virtual de aprendizagem. Anais do XXVIII Congresso da SBC.

VASCONCELOS, F. H. L., SILVA, T. E. V., GOMES, P. R. B., NUNES, A. O., ANDRIOLA, W. B., ALMEIDA, A. L. F., MOTA, J. C. M. (2011) Análise do Desempenho Discente em um Ambiente Virtual de Aprendizagem Através de Decomposições Tensoriais Multilineares. In: Anais do XXII SBIE - XVII WIE.

SPSS (2007). SPSS Statistics Base 17.0 User's Guide. Inc SPSS. 